\title{
Análisis del estilo de gestión del conflicto interpersonal en estudiantes universitarios
}

\section{Analysis of the management style of interpersonal conflict in university students}

\section{$\mathbf{M}^{\mathrm{a}}$ del Carmen Pegalajar Palomino}

Universidad de Jaén (UJA), España

\section{Resumen}

Esta investigación analiza el estilo de afrontamiento del conflicto interpersonal en estudiantes noveles del Grado de Educación Infantil de la Universidad de Jaén $(n=251)$. A partir de una investigación cuantitativa-correlacional, se utiliza el cuestionario "Rahim Organizational Conflict Inventory" (ROCI-II). Los estudiantes utilizan preferentemente estrategias basadas en la acomodación, solución de problemas y aplazamiento para la resolución del conflicto entre iguales, demostrándose relaciones de correspondencia entre ellas. Las mujeres obtienen mejores puntuaciones en los estilos de "Integración" y "Servilismo", mientras que los que mantienen la misma distribución que en otras asignaturas utilizan estilos basados en la "Evitación" y el "Servilismo".

Palabras clave: Educación Superior; conflicto; relaciones interpersonales; estudiantes.

\section{Abstract}

This research analyzes the coping style of interpersonal conflict in new students of the Degree in Early Childhood Education of the University of Jaén $(n=251)$. Based on a quantitative-correlational investigation, the questionnaire "Rahim Organizational Conflict Inventory" (ROCl-II) is used. Students preferably use strategies based on accommodation, problem solving and postponement for the resolution of conflict between equals, demonstrating correspondence relationships between them. The women obtain better scores in the styles of "Integration" and "Servilism", while those who maintain the same distribution as in other subjects use styles based on "Avoidance" and "Servilism".

Keywords: Higher education; conflict; interpersonal relationships; students.

\section{INTRODUCCIÓN}

En la última década, se asiste un proceso de transformación en Educación Superior, destacándose la rápida expansión tanto en el volumen de estudiantes como en las reformas que implican a las organizaciones de enseñanza universitaria (Goodwill, 2012). El nuevo espacio creado apuesta por una renovación metodológica, accediendo a un enfoque didáctico que otorga mayor importancia al aprendizaje del estudiante (Romero, Zurita-Ortega y Zurita-Molina, 2010), encaminado no sólo a su empleabilidad sino al desarrollo de un proceso de formación integral (Silva, 2016). 
De este modo, se apuesta por el desarrollo de metodologías innovadoras basadas en el análisis de casos, el aprendizaje basado en problemas, el aprendizaje basado en proyectos, el aprendizaje cooperativo y reflexivo, entre otros (Cañabate, Aymerich, Falgás y Gras, 2014; Gámez y Torres, 2012). Estas contribuyen a la mejora del proceso de enseñanza-aprendizaje, así como también favorecen el clima de aula, la cooperación, la pertenencia al grupo, la cohesión, el respeto y la empatía entre los estudiantes.

Según Vallet-Bellmunt, Rivera, Vallet-Bellmunt, y Vallet-Belmunt (2017), el aprendizaje cooperativo está basado en varios postulados, los cuales ofrecen los referentes básicos para que se produzca el aprendizaje en el estudiante: interacción con los compañeros (teoría cognitiva y teoría de la interdependencia social), interacción con el profesor (teoría comportamental), compromiso (teoría de interdependencia social) y aprendizaje activo (teoría cognitiva).

Este nuevo modelo formativo potencia la interdisciplinariedad, el trabajo en grupo, los procesos de búsqueda y selección de información, la construcción personal del aprendizaje, así como la implicación y adquisición de las capacidades personales del estudiante. Se trata de habilidades cognitivas, sociales, emocionales y éticas (iniciativa, esfuerzo por la calidad, responsabilidad, etc.) que tienen carácter transferible y constituyen el "saber ser" de la educación profesional en el estudiante universitario (Imaz, 2015).

Se busca, mediante esta nueva metodología, la necesidad de formar al estudiante para pensar, decidir y actuar en la vida real (Vallejo y Molina, 2014); se pretende dirigir los aprendizajes teóricos y prácticos a la formación para la vida, centrándolos en el desarrollo de competencias (Díaz, 2014). Dentro del marco del Espacio Europeo de Educación Superior, el proyecto "Tuning Educational Structures in Europa Project" (2009), describe que las competencias transversales pueden tener un carácter instrumental, sistémico e interpersonal, estando estas últimas relacionadas con las habilidades comunicativas y críticas, que favorecen la buena interacción del estudiante con los demás. Estas pueden tener un carácter individual (relativas a la capacidad de expresar los sentimientos, habilidades críticas y de autocrítica) o social (relacionadas con la capacidad de trabajar en equipo, expresión de compromiso social o ético).

Las competencias interpersonales están marcadas por su carácter actitudinal, resultando esenciales para la formación y construcción de futuros profesionales que deberán trabajar en equipo y formar parte activa dentro de una organización 
(Navarro, López, Climent y Ruiz, 2012). Entre ellas, se encuentran la gestión de conflictos, entendida como la capacidad para resolver de manera eficaz cualquier situación, hecho o problema que afecte a las relaciones interpersonales y/o ponga en riesgo los objetivos, intereses y necesidades del estudiante, así como la capacidad de negociación, a través de la cuál es posible la descripción de acuerdos a partir de diferentes tácticas.

Tomando como referencia lo anterior, se plantea el objeto de estudio de esta investigación a partir de la conceptualización del término "conflicto". Éste surge de la premisa de que se trata de un fenómeno connatural a la existencia humana (Silva, 2008; Maliandi, 2010), presentando un carácter transversal para todo tipo de interacciones y relaciones sociales. Según Osorio (2012) dicho concepto debe definirse como:

[...] una situación en la que un actor (persona, comunidad, Estado...) percibe que uno o varios de sus fines, propósitos, preferencias o medios para alcanzarlos es amenazado o estorbado por las intenciones o actividades de uno o varios de los demás actores con que interactúa (del mismo o de diferente rango), lo que conduce a una oposición, enfrentamiento o lucha. (p.64).

Por su parte, los estilos para el manejo de los conflictos deben entenderse como la manera en que la persona se aproxima a la otra parte ante una situación problemática. En este sentido, Rahim y Bohoma (1979) establecen una reinterpretación de los estilos de manejo del conflicto, diferenciándolos según dos dimensiones: interés propio e interés de los otros. La primera explica la medida en que un determinado sujeto satisface su propio interés, mientras que la segunda describe el procedimiento en que un individuo quiere o satisface el interés de los demás. Desde esta perspectiva, los cinco estilos posibles para el manejo del conflicto quedan distribuidos según:

- Integración: implica la colaboración entre los actores, lo que supone apertura, intercambio de información y análisis de las diferencias existentes para llegar a una solución aceptada por ambas partes.

- Servilismo: no considera las diferencias existentes con la otra parte, enfatizando y destacando los aspectos comunes entre ambos para procurar satisfacer el interés el otro.

- Dominación: procura por todos los medios lograr su objetivo y, como consecuencia, ignorar las necesidades y expectativas del otro.

- Evitación: asociado a la retirada y esquivación del problema, intenta posponerlo hasta un momento más propicio. 
- Compromiso: las partes ceden algo en su posición para tomar una decisión mutuamente aceptada para ambas.

El trabajo de campo planteado en esta investigación toma como referencia la importancia de gestionar adecuadamente el conflicto interpersonal entre estudiantes universitarios, aproximándose a la otra parte en base al desarrollo de unas adecuadas habilidades y competencias interpersonales que le permitan la adecuada gestión y la mejora de su rendimiento académico en los estudios que realizan.

El análisis del actual contexto universitario permite determinar cómo los adolescentes reconocen los conflictos a modo de resultados derivados de dificultades de la convivencia, tendiendo a calificar dicha situación con normalidad (Delpino, 2011), e incluso identificar su potencial constructivo. En este sentido, autores como Peterson (2014) y Arias-Cardona y Arias-Gómez (2017) entienden que los conflictos también pueden resultar beneficiosos en las organizaciones eficaces; estos autores aluden a los aspectos positivos del conflicto entre jóvenes estudiantes, entre los que destacan su dimensión transformadora y dinamizadora de los procesos sociales.

Su origen se asocia a las tensiones dialógicas relacionadas con la autonomía, la revelación de información sensible o la predictibilidad (García, 2015). Por su parte, y según Jaramillo, Arias-Cardona, Arias-Gómez, Restrepo y Ruiz (2012), los orígenes del conflicto entre estudiantes de Educación Superior pueden deberse a causas relacionadas con asuntos académicos, elementos personales, relaciones entre mujeres y dificultades entre subgrupos; según estos autores, el conflicto entre estudiantes universitarios es interpretado como violencia física, siendo los asuntos académicos la principal dificultad que encuentran en sus relaciones interpersonales. Por su parte, Benevides, Ribeiro y Da Silva, De Almeida-Lima, De Abreu y De AlmeidaSantos (2015) analizan las percepciones de los estudiantes universitarios sobre las relaciones interpersonales, destacándose un desempeño social insatisfactorio entre los miembros de la comunidad académica y, más concretamente, entre estudiantes y alumno/profesor.

La revisión de la literatura sobre el tema objeto de estudio revela la escasa bibliografía existente sobre el análisis de la gestión del conflicto entre estudiantes universitarios, llevándose la mayor parte de los estudios en el ámbito internacional. No obstante, cabe destacar algunas investigaciones como la propuesta por Navarro et al. (2012) quiénes analizan el perfil de estilos de gestión conductual de los conflictos en estudiantes adscritos a las titulaciones de Derecho y Psicología de la Universidad de Huelva. Sus resultados evidencian el predominio de los estudiantes 
en el afrontamiento del conflicto de manera integrativa y comprometida, lo que implica su tendencia a solucionar los conflictos buscando acuerdos que satisfagan los intereses propios y los de los demás. Estos resultados concuerdan con los aportados en la investigación desarrollada por Villamediana, Donado y Zerpa (2015), quiénes revelaron cómo los estudiantes universitarios suelen hacer uso del estilo integrador, seguido del comprometido, dominando y, finalmente, el evitativo para la gestión del conflicto.

Centrados en el análisis para la gestión del conflicto en los futuros docentes, cabe destacar estudios como los desarrollados por Peñalva, López y Landa (2013) en el contexto nacional. Así pues, se ha demostrado cómo uno de cada dos alumnos presenta un déficit en el desarrollo de las competencias emocionales que incluyen habilidades personales e interpersonales, siendo las habilidades en esta área superiores en relación a la población universitaria (Bueno, Teruel y Valero, 2005). Incluso, Pertegal, Castejón y Martínez (2009) muestran que los estudiantes no están preparados para una integración laboral exitosa en cuanto a competencias emocionales se refiere; no tienen las habilidades suficientes para trabajar en equipo, manejar con personas, adaptarse a cambios continuos o controlar sus emociones, entre otras.

Por su parte, distintos estudios (Garaigordobil, Machimbarrena y Maganto, 2016; Laca, Mejía y Mayoral, 2011; Luna y Laca, 2014; Luna y De Gante, 2017) aluden a los posibles efectos de variables como el género del estudiante sobre los estilos de gestión de los conflictos. Ello puede deberse a las diferencias en los procesos de socialización diferencial según los roles y estereotipos de género tradicionales, lo que induce a considerar que las mujeres están siendo socializadas hacia el desarrollo de habilidades interpersonales en mayor grado que los varones.

Ante esta situación, el sistema universitario español se plantea como objetivo prioritario la mejora de la calidad de las relaciones interpersonales entre los jóvenes universitarios, favoreciendo el bienestar psicosocial de los estudiantes en relación con sus compañeros (Soares, Guisando, Almeida y Páramo, 2008). Por ello, cobra especial importancia el diseño de programas de promoción del bienestar psicológico en el estudiante como medio, a la vez, de mejorar la calidad de la enseñanza universitaria (Salami, 2010).

Según Livia y García (2015), el contexto educativo representa un lugar privilegiado para la promoción de las habilidades y el desarrollo de competencias socioemocionales, las cuáles contribuyen de forma positiva al bienestar personal y social de 
las personas. En este sentido, debe hacerse referencia al desarrollo de propuestas innovadoras como las desarrolladas por Vázquez y López (2016) a partir del diseño de un taller de gestión de conflictos entre estudiantes universitarios mediante una dinámica de Role Playing. La puesta en práctica de esta iniciativa revela cómo los estudiantes disponen de capacidades para solucionar satisfactoriamente los conflictos planteados, demostrándose una relación directa entre el estilo de gestión del conflicto utilizado, la circunstancia de situación y su personalidad.

Además, debe tenerse en cuenta cómo el ingreso del estudiante en el sistema universitario implica un reto para dicha organización, pues la guía que se ofrezca al estudiante durante este tiempo va a marcar el éxito académico y la culminación satisfactoria de éste en el ciclo formativo (Londoño, 2009). El éxito en el rendimiento académico de estudiantes universitarios está atribuido al estilo de aprendizaje, así como a la motivación intrínseca y extrínseca derivada de elementos de la personalidad, factores emocionales, habilidades y hábitos en el estudiante, así como aspectos contextuales dependientes del ambiente externo y que facilitan el desarrollo de tareas académicas (Maris y Noriega, 2013).

Así pues, el análisis del clima organizacional en el ámbito académico incluye varias dimensiones, entre las que se encuentra la socio-afectiva, encargada del análisis de las percepciones sobre los miembros de la institución respecto a las relaciones interpersonales y la satisfacción del estudiante con el contexto (Bermúdez, Pedraza y Rincón, 2015), estrechamente vinculado con el objeto de estudio de este trabajo.

\section{METODOLOGÍA}

\subsection{Objetivos de la investigación}

Esta investigación pretende identificar el estilo de afrontamiento y gestión del conflicto interpersonal en estudiantes de primer curso del Grado en Educación Infantil de la Universidad de Jaén. Al tratarse de estudiantes noveles, que en su mayor parte acceden por primera vez al sistema universitario, se considera de interés explorar este ámbito para la mejora de la calidad en Educación Superior.

Así pues, y de modo más concreto, se pretende conseguir los siguientes objetivos específicos:

- Determinar el perfil socio-demográfico para la población objeto de estudio. 
- Identificar los estilos de afrontamiento en estudiantes noveles universitarios ante conflictos interpersonales con sus compañeros.

- Analizar el grado de correspondencia entre las escalas del cuestionario, así como entre aquellos ítems que resultan más significativos para el análisis de los estilos de afrontamiento ante conflictos interpersonales entre estudiantes universitarios.

- Analizar la existencia de diferencias significativas a nivel estadístico en cuanto a los estilos de afrontamiento de los conflictos interpersonales entre estudiantes universitarios en función del género.

- Examinar diferencias estadísticamente significativas para los estilos de afrontamiento de los conflictos interpersonales entre estudiantes universitarios según el motivo de elección entre los miembros del grupo.

Así pues, y a partir de estos objetivos, se han planteado las siguientes hipótesis de trabajo:

- El estudiante novel universitario utiliza preferentemente estrategias de afrontamiento de conflictos interpersonales basadas en el interés del otro.

- Existe correspondencia entre los estilos de gestión del conflicto interpersonal entre estudiantes universitarios.

- Determinados factores sociodemográficos como el género o el motivo de elección para los miembros del grupo guardan relación con los estilos de afrontamiento de conflictos interpersonales entre iguales.

\subsection{Posicionamiento metodológico y técnica de recogida de datos}

Para dar respuesta a los objetivos anteriormente explicitados, se ha planteado una investigación basada en un diseño metodológico de carácter cuantitativocorrelacional, la cual utiliza la técnica de la encuesta como método principal de recogida de datos. Además, cabe destacar cómo se ha tenido en cuenta el objeto de estudio de esta investigación, así como las características de la muestra objeto de estudio para adecuar el proceso de investigación a dichas circunstancias.

Así pues, se ha utilizado como instrumento de recogida de datos la versión española del cuestionario "Rahim Organizational Conflict Inventory" (ROCI-II), el cuál ha sido adaptado y validado por Munduate, Ganaza y Alcaide (1993). Se ha optado por la utilización de un solo instrumento pues se trata de un estudio inicial que pretende conocer el punto de partida en estudiantes del Grado de Educación Infantil 
de la Universidad de Jaén hacia el afrontamiento del conflicto interpersonal. La dificultad para acceder a la muestra objeto de estudio y potenciar su participación en trabajos de investigación suponen la principal causa que justifica la existencia de un solo instrumento que arroje resultados al trabajo de campo planteado en la investigación. No obstante, el equipo investigador es consciente de la existencia de determinadas variables explicativas que pueden tener impacto sobre la variable objeto de estudio (sociodemográficas, rendimiento académico, expectativas ante los estudios, competencias sociales, habilidades interpersonales, etc.).

Dicho cuestionario presenta las formas A, B y C, correspondientes a relaciones con superiores, relaciones con subordinados y relaciones con compañeros. En nuestro caso, y dado que el objeto de estudio de la investigación es analizar la gestión del conflicto interpersonal entre estudiantes universitarios, se ha utilizado la forma $C$ (estilos de gestión del conflicto entre compañeros). Consta de 28 ítems y mide los estilos de gestión del conflicto según una escala tipo Likert con 5 puntos (siendo $1=$ nunca y $5=$ siempre):

1. Integrador: implica alto interés propio y alto interés por los otros (7 ítems).

2. Dominante: refleja alto interés propio y bajo interés por los otros (5 ítems)

3. Evitativo: muestra bajo interés propio y bajo por los demás ( 6 ítems).

4. Complaciente: revela bajo interés propio y alto interés por los demás (7 ítems).

5. Comprometido: implica interés intermedio propio y por los demás (3 ítems).

La versión inicial del instrumento centra su diseño en el feedback de los sujetos y el análisis factorial de los 35 ítems originales (7 para cada estilo). Dicho instrumento ha sido traducido y adaptado al castellano en sus tres formas. Así pues, y tras una primera traducción, se ha llevado a cabo una prueba de interpretación y comprensión entre varios ejecutivos. Posteriormente, y teniendo en consideración sus indicaciones, se modificaron los ítems en los que se detectaron errores y fallos de interpretación, desarrollándose un juicio de expertos para así comprobar la asignación de cada ítem al estilo de gestión pertinente según la versión original. Finalmente, el cuestionario ha sido distribuido entre 310 ejecutivos, quedando la muestra definitiva constituida por 226 directivos.

Las respuestas de los directivos permitieron llevar a cabo el análisis de fiabilidad del instrumento, obteniéndose un coeficiente alfa de Cronbach para la escala total de .78. Por su parte, la consistencia interna del instrumento adaptado también resulta satisfactoria, siendo la escala de "Integración" la que obtiene una mayor puntuación (.77), y la de "Compromiso" la que obtiene un valor más bajo (.62). Por su parte, el 
análisis de factores principales realizado revela la existencia de siete factores que explican el 57.2\% de la varianza. Estos factores coinciden con los anteriormente citados, siendo el sexto factor el que contiene dos ítems que en la escala original se vinculan a "Servilismo", mientras que el séptimo contiene un ítem que en la escala original pertenece a "Evitación".

\subsection{Muestra}

La población objeto de estudio está compuesta por estudiantes de primer curso del Grado en Educación Infantil de la Universidad de Jaén $(n=251)$. Por su parte, la muestra la conforman 226 estudiantes que participaron en la investigación de manera voluntaria a partir de la cumplimentación del cuestionario.

Para la selección de los sujetos se ha utilizado un muestreo probabilístico aleatorio simple, de forma que se han adscrito a la muestra todos aquellos sujetos que al azar respondieron a los cuestionarios enviados. Para el cálculo de la muestra participante se utilizó la fórmula para poblaciones con menos de 100.000 sujetos con un nivel de confianza del $95 \%$ y un error de estimación máximo de un 5\%.

De la muestra, un $85.7 \%$ está conformado por mujeres, frente al $14.3 \%$ que son hombres. Éstos poseen una edad inferior a los 20 años (50.5\%), frente al 48.5\% cuya edad se sitúa entre los 21 y 30 años y un $1.0 \%$ de estudiantes que son mayores de 31 años. En un $91.5 \%$ de los casos, son estudiantes matriculados en la asignatura "Didáctica General en Educación Infantil" en su primera convocatoria, frente al $8.5 \%$ de la muestra que confirma haber estado matriculado en dicha asignatura con anterioridad, por lo que están cursando esta asignatura en segunda o tercera convocatoria. Al cuestionar su grado de satisfacción respecto a grupo de trabajo desarrollado para la realización de las actividades prácticas propuestas, un 77.3\% la considera como "buena", mientras que un 22.7\% la califica de "regular". Finalmente, y en cuanto al motivo de elección entre los miembros del grupo, un $53.1 \%$ sostiene que ha conformado el grupo de trabajo a partir de una relación de amistad entre los miembros desarrollada durante los primeros días de clase, un $31.6 \%$ sostiene que ha elegido a los miembros de su grupo a azar, un 15.3\% por continuidad, al pertenecer a ese grupo de trabajo en otras asignaturas.

\subsection{Procedimiento}

El cuestionario se ha administrado a los estudiantes de manera individual, una vez se ha contado con el visto bueno de los docentes implicados en cada uno de los grupos de la asignatura "Didáctica General en Educación Infantil" adscrita al plan 
de estudios del Grado en Educación Infantil de la Universidad de Jaén. De este modo, y al tratarse de una asignatura de carácter anual que debe ser cursada por el estudiante durante el primer curso, se ha decidido estudiar la percepción del estudiante hacia la gestión de los conflictos interpersonales para, de este modo, adecuar el proceso de enseñanza-aprendizaje a tales circunstancias. Se debe destacar cómo, al ser estudiantes noveles que en su mayoría acceden por primera vez al sistema universitario, no existen apenas vínculos entre ellos, por lo que no se han desarrollado relaciones positivas ni negativas entre los estudiantes.

Así pues, y con la intención de responder a algunos de los objetivos y resultados de aprendizaje propuestos en la guía docente de la asignatura, el profesorado implicado en su docencia plantea la realización de un trabajo práctico de carácter grupal, siendo la metodología basada en el aprendizaje cooperativo la estrategia metodológica básica para el desarrollo del proceso de enseñanza-aprendizaje. De este modo, se han conformado grupos de trabajo compuestos por un máximo de cinco personas, tratando de fomentar la heterogeneidad entre sus integrantes (de género, capacidad, motivación), para así favorecer habilidades de cooperación, diálogo y reflexión compartida.

Por su parte, y para conseguir una correcta aplicación del instrumento, se ha informado a los estudiantes acerca de las normas de cumplimentación, características del cuestionario y finalidad de la misma asegurándole, en todo momento, el carácter anónimo y confidencial de los datos recogidos.

\subsection{Análisis de datos}

Tras la cumplimentación del cuestionario por el alumnado, se ha llevado a cabo el análisis de datos mediante el paquete informático Statistickal Packagefor Social Sciences (SPSS versión 22) al considerarlo un recurso idóneo para esta investigación. De este modo, se ha utilizado la estadística descriptiva (a partir del análisis de medias y desviaciones típicas), así como el coeficiente de correlación de Pearson para conocer el grado de relación entre las escalas del cuestionario, así como entre aquellos ítems considerados de interés según el objetivo de la investigación. Además, se realiza la prueba de comparación de medias $t$ de Student, con la intención de demostrar la existencia o no de diferencias significativas a nivel estadístico entre las escalas y la variable sociodemográfica "género", así como el análisis de varianza (ANOVA) para analizar la existencia de diferencias significativas a nivel estadístico entre las escalas y la variable sociodemográfica "motivación para la elección de los miembros del grupo". 


\section{RESULTADOS}

En primer lugar, se presentan las puntuaciones medias obtenidas por la muestra objeto de estudio para cada una de las dimensiones que componen el cuestionario "Rahim Organizational Conflict Inventory" (ROCl-II). Así pues, tal y como se muestra en la tabla 1, la puntuación más alta corresponde a la escala "Servilismo" ( $M=4.95$; DT=.580), seguida de "Integración" ( $M=4.54 ; D T=.631)$ y "Evitación" ( $M=3.95$; DT=.853), siendo las de menor puntuación la escala de "Compromiso" ( $M=3.06$; $D T=.953)$ y "Dominación" ( $M=2.59 ; \mathrm{DT}=.647)$. Ello indica cómo existe un mayor número de estudiantes que utilizan las estrategias basadas en el desarrollo de conductas de acomodación, integración y solución de problemas, así como las de aplazamiento para la resolución del conflicto entre sus iguales.

Así pues, y en relación a la escala "Servilismo", destacan los resultados obtenidos en el ítem 7 "Trato de encontrar caminos intermedios para avanzar hacia una solución" $(\mathrm{M}=4.57$; $\mathrm{DT}=$.656) y el ítem 2 "Ante un problema de trabajo, generalmente trato de satisfacer los deseos de mi compañero/a" (M=3.93; DT=.904). Por su parte, y para la escala "Integración", destacan los resultados obtenidos en el ítem 5 "Trato de analizar con mi compañero/a las soluciones a los problemas que nos beneficien a ambos" ( $M=4.63$; DT=.612) o el ítem 4 "Ante una situación problemática con mi compañero/a, intento integrar mis ideas y las suyas para alcanzar una decisión conjunta" ( $M=4.61$; DT=.548). Finalmente, y para la escala "Evitación", destacan los resultados obtenidos en el ítem 27 "Intento evitar situaciones enojosas y desagradables con mi compañero/a" (M=4.37; DT=.821), así como el ítem 17 "Evito tropiezos con mi compañero/a" (M=4.36; DT=.817).

TABLA 1

\section{Análisis descriptivo de los datos}

\begin{tabular}{lccc}
\hline & N & M. & D.T. \\
\hline Servilismo & 221 & 4.96 & .580 \\
Integración & 226 & 4.54 & .631 \\
Evitación & 213 & 3.95 & .853 \\
Compromiso & 223 & 3.06 & .953 \\
Dominación & 225 & 2.59 & .647 \\
\hline
\end{tabular}

Fuente: Elaboración propia.

Una vez realizado el análisis descriptivo de las escalas que incluyen el cuestionario, se analiza su grado de relación a partir del análisis de correlación r de Pearson (tabla 2). Los resultados obtenidos revelan cómo la mayor correspondencia se produce 
entre el estilo de gestión del conflicto interpersonal basado en el "Servilismo", en el que el estudiante muestra bajo interés personal y una alta preocupación por los demás, y el de "Evitación", basado en el bajo interés por los resultados propios y ajenos ( $r=.602)$. De igual modo, se aprecia correspondencia entre el estilo basado en el "Compromiso", quién adopta un estilo intermedio en interés propio y por los demás, y de "Integración", basado en el alto interés por uno mismo y los demás ( $r=.463)$. Este último guarda relación, además, con el estilo de gestión del conflicto basado en el "Servilismo" ( $r=.369)$. Finalmente, se destaca cómo existe correspondencia entre el estilo basado en el "Compromiso" y el de "Dominación", caracterizado por un alto interés personal y bajo interés por los demás ( $r=.351)$.

TABLA 2

\section{Correlación entre las distintas escalas del cuestionario}

\begin{tabular}{lccccc}
\hline & Integración & Dominación & Compromiso & Evitación & Servilismo \\
\hline Integración & & & $.463^{* *}$ & $.312^{* *}$ & $.369^{* *}$ \\
Dominación & & & $.351^{* *}$ & & $.200^{* *}$ \\
Compromiso & $.463^{* *}$ & $.351^{* *}$ & & $214^{* *}$ & $.326^{* *}$ \\
Evitación & $.312^{* *}$ & & $.214^{* *}$ & & $.602^{* *}$ \\
Servilismo & $.369^{* *}$ & $.200^{* *}$ & $.326^{* *}$ & $.602^{* *}$ & \\
\hline
\end{tabular}

** Nivel de significación $<.05$

Fuente: Elaboración propia.

Igualmente, se han analizado las relaciones de correspondencia entre algunos de los ítems más significativos para el análisis de esta investigación y entre los que figuran (tabla 3): V1: Ante una dificultad en el trabajo con mis compañeros, intento analizar la situación con él para encontrar una solución aceptable para ambos; V6: Cuando se trata de problemas de trabajo, generalmente evito discutir abiertamente con mi compañero/a; v8: Procuro utilizar todos los recursos a mi alcance para conseguir que mis ideas sean aceptadas; V10: Generalmente, actúo como desea mi compañero/a; V14: Generalmente, propongo un camino intermedio para romper con los puntos muertos.

Así pues, las mayores correlaciones se producen al cuestionar a los estudiantes la posibilidad de analizar la situación para encontrar una solución aceptable para ambas partes y proponer un camino intermedio para romper con los puntos muertos ante una situación de dificultad en el trabajo en grupo $(r=.352)$. Igualmente, se producen correlaciones al cuestionar al alumnado la posibilidad de romper con los puntos muertos ante una dificultad y el hecho de utilizar los recursos a su alcance para conseguir que las ideas propias sean aceptadas por la colectividad ( $r=.318)$. Finalmente, también se destaca la existencia de correspondencia al valorar la 
actuación del estudiante conforme a las exigencias y requisitos de su grupo de iguales y la eliminación de discusiones con los compañeros ante un problema de trabajo $(r=.317)$.

TABLA 3

\section{Correlación entre ítems más significativos del cuestionario}

\begin{tabular}{lccccc}
\hline & V1 & V6 & V8 & V10 & V14 \\
\hline V1 & & $.134^{*}$ & $.268^{* *}$ & .020 & $.352^{* *}$ \\
V6 & $.134^{*}$ & & .013 & $.317^{\star *}$ & .052 \\
V8 & $.268^{* *}$ & .013 & & $.158^{*}$ & $.318^{* *}$ \\
V10 & .020 & $.317^{* *}$ & $.158^{*}$ & & .124 \\
V14 & $.352^{* *}$ & .052 & $.318^{* *}$ & .124 & \\
\hline
\end{tabular}

*Nivel de significación $<.01$; ** Nivel de significación $<.05$

Fuente: Elaboración propia.

Por su parte, la prueba de diferencias de medias (prueba t de Student) ha permitido identificar la existencia de diferencias significativas a nivel estadístico entre los estilos de afrontamiento de los conflictos interpersonales entre estudiantes universitarios y la variable sociodemográfica "género". Las diferencias significativas a nivel estadístico se aprecian para las escalas "Integración" $\left(\mathrm{t}\left(\mathrm{z24}_{4}\right)=2.921\right.$, $\left.\mathrm{p}=.001\right)$ y "Servilismo" $\left(\mathrm{t}\left(_{219}\right)=2.895, \mathrm{p}=.005\right)$. No obstante, no se aprecian diferencias significativas a nivel estadístico para las escalas de "Dominación" $\left(\mathrm{t}\left({ }_{223}\right)=4.143, \mathrm{p}=.148\right)$, "Compromiso" $\left(\mathrm{t}\left(_{221}\right)=3.758, \mathrm{p}=.891\right)$ y "Evitación" $\left(\mathrm{t}\left(\mathrm{211}_{1}\right)=2.995, \mathrm{p}=.175\right)$.

Así pues, tal y como se aprecia en la figura 1, las mujeres obtienen mejores puntuaciones al cuestionar la búsqueda de soluciones para satisfacer las aspiraciones de todas las partes implicadas para el desarrollo del trabajo grupal, así como la puesta en práctica de conductas de acomodación con la intención de adaptarse a los intereses del otro llegando, incluso, a ceder ante sus demandas. Además, las valoraciones de los estudiantes suelen ser similares al cuestionar la puesta en práctica de conductas para conseguir fines propios sin tener en cuenta las opiniones, intereses y necesidades de los demás, la obtención de acuerdos satisfactorios para ambas partes, así como el aplazamiento para la resolución del conflicto. 
FIGURA 1

\section{Medias de las escalas según la variable "género" (Prueba $t$ )}

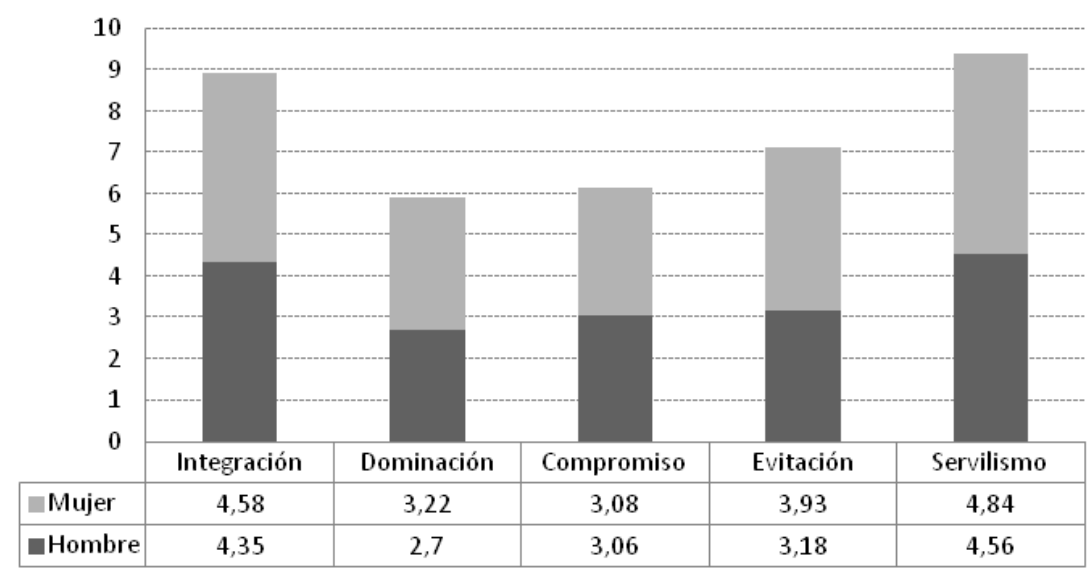

Fuente: Elaboración propia.

Finalmente, el análisis de varianza (ANOVA) permite determinar la existencia de diferencias significativas a nivel estadístico entre las escalas del cuestionario y la variable sociodemográfica "motivación para la elección de los miembros del grupo". Las diferencias en las valoraciones de los estudiantes se vinculan a la escala de "Evitación" $\left.\left(\mathrm{F}_{3,209}\right)=5.862, \mathrm{p}=.001\right)$, centrada en el aplazamiento para la resolución del conflicto que pueda surgir entre iguales. La prueba Tukey realizada a posteriori revela cómo dichas diferencias se sitúan entre los estudiantes que han elaborado los grupos de trabajo seleccionando a sus miembros al azar, y aquellos otros que lo han realizado para continuar la misma distribución grupal que en otras asignaturas, siendo las valoraciones más favorables para éstos últimos, tal y como se aprecia en la figura 2.

En segundo lugar, también se aprecian diferencias significativas a nivel estadístico para la escala "Servilismo" $\left.\left(\mathrm{F}_{3,217}\right)=2.284, \mathrm{p}=.005\right)$, centrada en la acomodación del estudiante a los intereses de los demás. En este caso, las diferencias a nivel estadístico se sitúan entre aquellos estudiantes que han configurado los grupos de trabajo al azar y aquellos otros que lo han hecho a partir de relaciones de amistad entre sus componentes, siendo las valoraciones más favorables para los primeros, siendo representada gráficamente en la figura 2.

No obstante, no se aprecian diferencias significativas a nivel estadístico para las escalas de "Integración" $\left(F\left({ }_{3,222}\right)=.851, p=.467\right)$, "Dominación" $\left(F\left({ }_{3,221}\right)=1.707, p=.167\right)$ y "Compromiso" $\left.\left(\mathrm{F}_{3,219}\right)=1.260, \mathrm{p}=289\right)$. 
FIGURA 2

Medias de las escalas según la variable "motivación para la elección de los miembros del grupo" (ANOVA)

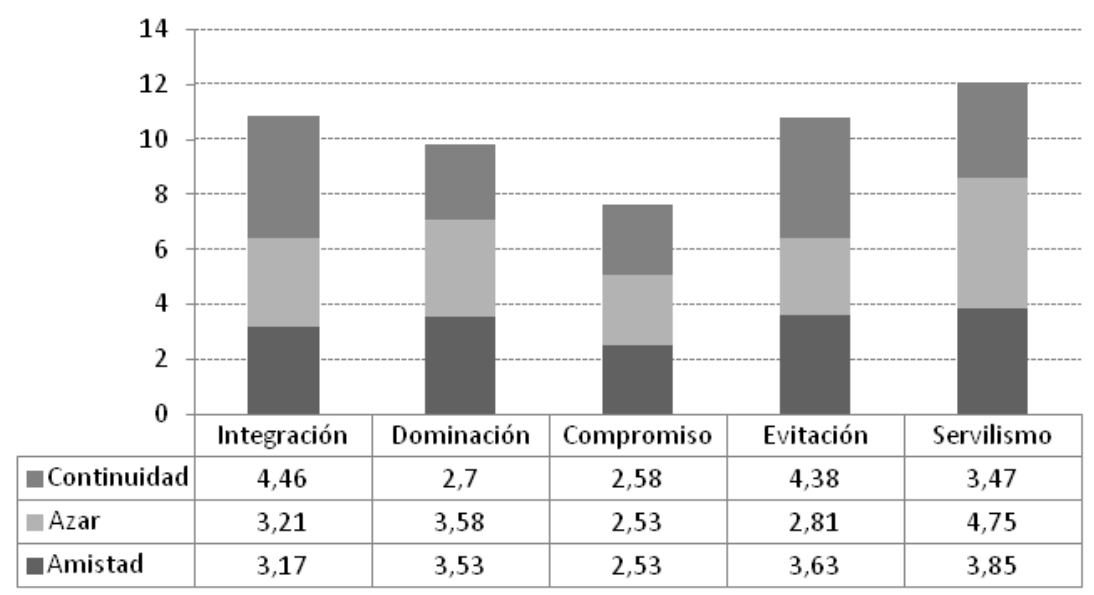

Fuente: Elaboración propia.

\section{DISCUSIÓN Y CONCLUSIONES}

Esta investigación ha permitido identificar el estilo de afrontamiento y gestión del conflicto interpersonal en estudiantes noveles adscritos al Grado en Educación Infantil de la Universidad de Jaén. Se trata de un trabajo de enorme interés, pues trata de explorar dicho ámbito para la mejora de la calidad educativa en el ámbito universitario.

En este caso, se ha optado por dicho análisis a partir del desarrollo de metodologías activas basadas en el aprendizaje cooperativo en Educación Superior, las cuales posibilitan el desarrollo de competencias interpersonales en el estudiante, entre otras (Díaz, 2014). El hecho de analizar el estilo de gestión del conflicto en las relaciones interpersonales entre estudiantes en Educación Superior va a permitir al profesorado universitario conocer cómo se relacionan sus estudiantes para el desarrollo del proceso de enseñanza-aprendizaje, así como el modo en que son capaces de resolver de manera eficaz cualquier situación problemática que afecte a las relaciones interpersonales y ponga en riesgo los intereses y necesidades del estudiante en Educación Superior. 
Los resultados demuestran que los estudiantes objeto de estudio de esta investigación hacen uso de estrategias para la resolución de situaciones problemáticas en el seno del trabajo cooperativo a partir del desarrollo de conductas de acomodación y servilismo para satisfacer el interés del otro, integración y solución de problemas con las que llegar a una solución aceptada por ambas partes y de aplazamiento y esquivación para la resolución del conflicto entre sus iguales.

Estos resultados concuerdan con los aportados en las investigaciones desarrolladas por Navarro et al. (2012) o Villamediana, Donado y Zerpa (2015), quiénes aluden al estilo integrador como modelo predominante en el afrontamiento del conflicto entre estudiantes universitarios. Se trata de un estilo que adopta la colaboración entre sus miembros para el intercambio de información y análisis de las diferencias existentes con las que llegar a un acuerdo aceptado por ambas partes. Ello denota la motivación y el interés del estudiante hacia el desarrollo de los trabajos prácticos a partir del aprendizaje cooperativo, lo cual repercute de forma directa en la formación y construcción de los futuros docentes que deberán trabajar en equipo y formar parte activa de la estructura organizacional del contexto educativo en que desarrollar su actividad docente (Navarro et al., 2012).

24 No obstante, también cabe destacar el desarrollo de estilos basados en el servilismo y la satisfacción de los deseos del otro ante una situación problemática, así como las de aplazamiento y/o esquivación del problema ante el conflicto con sus iguales. Estos resultados revelan el déficit en el desarrollo de competencias emocionales para estudiantes de Magisterio que subrayan las investigaciones propuestas por Peñalva, López y Landa (2013) o Bueno, Teruel o Valero (2005). Además, debe tenerse en cuenta las carencias formativas de los estudiantes de Magisterio para el desarrollo de propuestas metodológicas basadas en el aprendizaje cooperativo, el manejo con personas, la adaptación a cambios continuos o el control emocional que propone el estudio de Pertegal, Castejón y Martínez (2009).

Teniendo en cuenta las diferencias en los estilos de afrontamiento de los conflictos interpersonales según el género del estudiante, cabe destacar cómo las mujeres obtienen mejores puntuaciones para la escala de "integración" y "servilismo". Así pues, los estudiantes de género femenino son capaces de desarrollar mejores estrategias basadas en la búsqueda de soluciones con las que satisfacer las aspiraciones de todas las partes implicadas en el trabajo cooperativo, así como la puesta en práctica de acciones para la adaptación a los intereses del otro llegando, incluso, a ceder ante sus demandas. 
Estos resultados guardan relación con los resultados de las investigaciones propuestas por Garaigordobil, Machimbarrena y Maganto (2016), Laca, Mejía y Mayoral (2011), Luna y Laca (2014) o Luna y De Gante (2017), quiénes aluden a los efectos de los estilos de gestión de los conflictos interpersonales sobre el género. Ello puede deberse a la influencia de los modelos educativos diferenciales sobre los estudiantes según el género, en las que se ha atribuido mayores esfuerzos para el desarrollo de habilidades interpersonales para las mujeres. Además, y en este caso, debe hacerse mención al concepto de "liderazgo femenino" dentro de la cultura organizacional y que aparece clasificado según (Díez, Terrón y Anguita, 2006):

- Diferencias interpersonales, entre las que se destacan la capacidad para trabajar en equipo (dirección participativa centrada en el grupo), mejores habilidades de comunicación (saber escuchar y empatía), creación de un clima de bienestar basado en el diálogo y la escucha, liderazgo compartido basado en la cooperación y el trabajo en equipo, resolución de conflictos basado en la promoción del diálogo, la tolerancia y comprensión de las situaciones conflictivas.

- Diferencias intrapersonales, basadas en la capacidad multimental para conjugar adecuadamente la intuición, emocionalidad y racionalidad en la toma de decisiones, el desarrollo profesional del estudiante, que tiende a involucrarse en proyectos y trabajos de innovación y mejora, así como la constancia profesional para conseguir objetivos a largo plazo.

Finalmente, y en cuanto a la variable sociodemográfica que trata de analizar el "motivo del estudiante para la elección de los miembros del grupo", se aprecian diferencias estadísticas para los estudiantes que responden al conflicto interpersonal a partir de estilos basados en la "evitación" y el "servilismo", siendo más favorables para aquellos que han seleccionado a sus miembros con la intención de continuar la misma distribución grupal que en otras asignaturas o al azar, frente a aquellos otros que han preferido destacar las relaciones de amistad entre sus miembros para configurar sus grupos de trabajo.

Así pues, y de modo razonable, cabe entender que aquellos estudiantes que han preferido continuar con el mismo grupo de trabajo que en otras asignaturas opten por esquivar el problema y/o la situación conflictiva con su grupo de iguales para así no perjudicar a los demás o salir perjudicado en la realización de los trabajos prácticos propuestos por el profesor. Por su parte, aquellos otros estudiantes que han seleccionado a sus miembros al azar tratan de ceder ante sus iguales, probablemente por el déficit que presentan hacia el desarrollo de habilidades interpersonales, 
así como también puede deberse a la falta de motivación para la realización de los trabajos grupales planteados por el profesor, así como la falta de interés ante los estudios universitarios que se encuentran realizando.

De este modo, investigaciones como las desarrolladas por Korkmaz y Yesil (2011) o Nam y Zellner (2011) destacan cómo gran parte del alumnado también se muestra reacio a participar en procesos de aprendizaje cooperativo, encontrándose en los que parte del grupo elude sus responsabilidades, poniendo en peligro el éxito de este tipo de aprendizaje (Korkmaz, 2012). En este sentido, Pujolás (2008) define a un grupo como cooperativo cuando se establece un objetivo común, relación de igualdad, interdependencia positiva, cooperación, ayuda y exigencia mutua entre todos los miembros, así como la existencia de un vínculo afectivo que hace que celebren juntos los éxitos conseguidos entre todos los miembros.

En definitiva, y modo de síntesis, se destaca el carácter integrador y colaborador de los estudiantes universitarios hacia la gestión de situaciones conflictivas en sus relaciones interpersonales con su grupo de iguales. No obstante, cobra importancia el diseño y desarrollo de programas para la promoción y mejora de las habilidades interpersonales en el estudiante universitario contribuyendo de este modo a la mejora del rendimiento académico del estudiante en Educación Superior.

Así pues, y como posibles limitaciones del estudio, cabe destacar que se trata de un estudio basado únicamente en el análisis de los estilos de gestión y afrontamiento del conflicto en estudiantes del Grado de Educación Infantil de la Universidad de Jaén, lo que impide la generalización de los resultados para otras muestras. De igual modo, el uso del cuestionario como instrumento de recogida de datos puede provocar problemas de deseabilidad social entre los encuestados.

Por ello, y de cara a futuras investigaciones, se pretende ampliar la muestra, accediendo a estudiantes otros Grados y/o facultades, tanto de la Universidad de Jaén, como de otras instituciones de Educación Superior tanto de carácter público como privado, con la intención de establecer comparativas entre las percepciones de los estudiantes para ambas muestras. Además, se pretende ampliar la metodología de la investigación, llevando a cabo análisis cualitativos basados en entrevistas y grupos de discusión que permitan analizar en profundidad el objeto de estudio de este trabajo. 


\section{REFERENCIAS BIBLIOGRÁFICAS}

Arias-Cardona, A.M. y Arias-Gómez, M. (2017). Conflicto y Educación Superior: narrativas y vivencias de jóvenes universitarios estudiantes de Ciencias Sociales y Humanas. Revista CES Psico, 11(1), 56-78.

Benevides, A., Ribeiro, A.M., Da Silva, A.M., De Almeida-Lima, C., Abreu, T. y Almeida-Santos, Z. (2015). Percepciones de los estudiantes de Psicología sobre las relaciones interpersonales en el ámbito universitario: un estudio exploratorio. Revista Diversitas. Perspectivas en Psicología, 11(1), 67-78.

Bermúdez, J. J., Pedraza, A. y Rincón, C. I. (2015). El clima organizacional en universidades de Bogotá desde la perspectiva de los estudiantes. Revista Electrónica de Investigación Educativa, 17(3), 1-12. Disponible en: http://redie.uabc.mx/ vol17no3/contenido-bermudezetal.html (consultado el 10 de Mayo de 2018).

Bueno, C., Teruel, M. P. y Valero, A. (2005). La inteligencia emocional en alumnos de Magisterio: la percepción y comprensión de los sentimientos y las emociones. Revista Interuniversitaria del Profesorado, 54, 169-194.

Cañabate, D., Aymerich, M., Falgás, M. y Gras, M. E. (2014). Metodologías docentes. Motivación y aprendizaje percibidos por los estudiantes universitarios. Educar, 50(2), 427-441.

Delpino, M.A. (2011). Inmigración y educación. El conflicto en la escuela española y sus percepciones. Tesis doctoral. Universidad de Salamanca, Departamento de Teoría e Historia de la Educación, Salamanca (España).

Díaz, A. (2014) Construcción de programas de estudio en la perspectiva del enfoque del desarrollo de competencias. Perfiles Educativos, 143, 142-162.

Díez, E.J., Terrón, E. y Anguita, R. (2006). La cultura de género en las organizaciones escolares. Motivaciones y obstáculos para el acceso de las mujeres a los puestos de dirección. Barcelona: Octaedro.

Gámez, M.J. y Torres, C. (2012). Las técnicas de grupo como estrategia metodológica en la adquisición de la competencia de trabajo en equipo de los alumnos universitarios. Journal form Educators, Teachers and Trainers, 4, 14-25.

Garaigordobil, M. y Maganto, C. (2011). Empatía y resolución de conflictos durante la infancia y la adolescencia. Revista Latinoamericana de Psicología, 43(2), 255-266.

Garaigordobil, M., Machimbarrena, J. M. y Maganto, C. (2016). Adaptación española de un instrumento para evaluar la resolución de conflictos Conflictalk): datos psicométricos de fiabilidad y validez. Revista de Psicología Clínica con Niños y Adolescentes, 3(2), 59-67.

García, L. (2015). La teoría de la comunicación como matriz práctica para la resolución de conflictos. Comunicación y Sociedad, 23, 45-65.

Goodwill, R. (2012). Engaging staffa communities in a knowledge transfer strategy: A case of study at the University of Melbourne. Journal of Higher Education Policy 
and Management, 34(3), 285-294. Disponible en: http://dx.doi.org/10.1080/1 360080X.2012.678726 (consultado el 15 de Mayo de 2018).

Imaz, J.I. (2015). Aprendizaje basado en proyectos en los grados de Pedagogía y Educación Social: ¿Cómo ha cambiado tu ciudad? Revista Complutense de Educación, 26(3), 679-696.

Jaramillo, C., Arias-Cardona, A., Arias-Gómez, M., Restrepo, F. y Ruiz, D. (2012). Relaciones sociales entre jóvenes universitarios: Una mirada desde sus lógicas subjetivas. Envigado: Institución Universitaria de Envigado.

Korkmaz, Ö. (2012). A validity and reliability study of the Online Cooperative Learning Attitude Scale (OCLAS). Computers \& education, 59, 1162-1169.

Korkmaz, Ö. y Yesil, R. (2011). Evaluation of achievement, attitudes towards technology using and opinions about group work among students working in gender based groups. Gazi University Journal of Gazi Education Faculty, 31(1), 201-229.

Laca, F.A., Mejía, J.C. y Mayoral, E.G. (2011). Conflict Communication, Decision-Making, and Individualism in Mexican and Spanish University Students. Psychology Journal, 8(1), 121-135.

Livia, G. y García, L. (2015). Estudio de las competencias socio-emocionales y su relación con el afrontamiento en futuros profesores de nivel medio. REIFOP. Revista Electrónica Interuniversitaria de Formación del Profesorado, 18(1), 213-228.

28 Londoño, C. (2009). Optimismo y salud positive como predictores de la adaptación a la vida universitaria. Acta Colombiana de Psicología, 12(1), 95-107.

Luna, A. y Laca, F. (2014). Estilos de mensajes en el manejo de conflictos en adolescentes y jóvenes mexicanos. Boletín de Psicología, 110, 37-51.

Luna, A.C. y De Gante, A. (2017). Empatía y gestión de conflictos en estudiantes de secundaria y bachillerato. Revista de Educación y Desarrollo, 40, 27-37.

Maliandi, R. (2010). Ética Convergente. Fenomenología de la conflictividad. Buenos Aires: Las Cuarenta. Disponible en: http://aaieticas.org/revista/index.php/cde/article/ view/23/48 (consultado el 10 de Mayo de 2018).

Maris, S. y Noriega, M. (2013). Relaciones entre rendimiento académico, competencia espacial, estilos de aprendizaje y deserción. Revista Electrónica de Investigación Educativa, 15(1), 29-44.

Munduate, L.; Ganaza, J. y Alcaide, M. (1993). Estilos de gestión del conflicto interpersonal en las organizaciones. Revista de Psicología Social, 8, 47-68.

Nam, C. W. y Zellner, R. D. (2011). The relative efects of positive interdependence and group processing on student achievement and attitude in online cooperative learning. Computers \& Education, 56, 680-688. doi: http://dx.doi.org/10.1016/j. compedu.2010.10.010 
Navarro, Y., López, Ma J., Climent, J.A. y Ruíz, Ma J. (2012). Desarrollo de competencias de gestión de conflictos en la formación universitaria. Revista Iberoamericana de Educación, 60(2), 1-11. Disponible en: https://rieoei.org/RIE/article/view/1322

Osorio, S. (2012). Conflicto, Violencia y Paz: Un acercamiento científico, filosófico y bioético. Revista Latinoamericana de Bioética, 12(2),052-069. Disponible en: http://www. redalyc.org/articulo.oa?id=127025833006 (consultado el 7 de Mayo de 2018).

Peñalva, A., López, J.J. y Landa, N. (2013). Competencias emocionales del alumnado de Magisterio: posibles implicaciones profesionales. Revista de Educación, 362, 690-712. DOI: 10.4438/1988-592X-RE-2013-362-246

Pertegal, M., Castejón, J. y Martínez, M. (2011). Competencias socioemocionales en el desarrollo profesional del maestro. Educación XX1, 14 (2), 237-260.

Peterson, J. (2014). Why conflict is good for business. Stanford Business School. Disponible en: https://www.linkedin.com/pulse/20140328060449-11846967-why-conflictis-good-for-business. (consultado el 10 de Mayo de 2018).

Pujolás, P. (2008). El aprendizaje cooperativo: 9 ideas clave. Barcelona: Graó.

Rahim, M. A. y Bonoma, T. V. (1979). Managing Organizational Conflict: A Model for Diagnosis and Intervention. Psychological Reports, 16, 143-155.

Romero, C., Zurita-Ortega, F. y Zurita-Molina, F. (2010). Autonomía y orientación en el Espacio Europeo de Educación Superior mediante el portafolio y la tutoría. ESE. Estudios sobre educación, 19, 261-282.

Salami, S.O. (2010). Emotional intelligence, self-efficacy, psychological well-being and students' attitudes: implications for quality implications. European Journal of Educational Studies, 2(3), 247-257.

Silva, G. (2008). La teoría del conflicto. Un marco teórico necesario. Prolegómenos. Derechos y Valores, 11(22), 29 - 43. Disponible en: http://www.redalyc.org/articulo. oa?id=87602203 (consultado el 7 de Mayo de 2018).

Silva, M. (2016). Competencias de estudiantes iberoamericanos al finalizar la educación secundaria alta. Perfiles Educativos, 38(152), 88-108.

Soares, A.P., Guisande, A.M., Almeida, L.S. y Páramo, F.M. (2008). Academic achievement in first-year portuguese college students: The role of academic preparation and learning strategies. Internacional Journal of Psychology, 44, 204-212.

Tuning, P. (2009). Tunning Educational Structures in Europe. Disponible en: http://www. unideusto.org/tuningeu/images/stories/Publications/Tuning brochure en espanol listo.pdf. (consultado el 9 de Junio de 2018).

Vallejo, M. y Molina, J. (2014). La evaluación auténtica de los procesos educativos. Revista Iberoamericana de Educación, 64, 11-25. Disponible en: https://rieoei.org/RIE/ article/view/403 
Análisis del estilo de gestión del conflicto interpersonal en estudiantes universitarios...

Vallet-Bellmunt, T., Rivera, P., Vallet-Bellmunt, I. y Vallet-Belmunt, A. (2017). Aprendizaje cooperativo, aprendizaje percibido y rendimiento académico de la enseñanza de marketing. Educación XX1, 20(1), 277-297.

Vázquez, M. y López, M.A. (2016). La gestión constructiva de conflictos. Propuesta y desarrollo de un taller práctico en el contexto universitario. Revista de Investigación Educativa, 14(2), 184-204.

Villamediana, J., Donado, A. y Zerpa, C.E. (2015). Estilos de manejo de conflictos, inteligencia emocional y desarrollo moral. Revista Dimensión Empresarial, 13(1), 73-94. 